\title{
FREE PRODUCTS OF LATTICE-ORDERED GROUPS
}

\author{
A. M. W. GLASS
}

Dedicated to Sydney Berlanny-in memoriam

\begin{abstract}
We give a highly homogeneous representation for the free product of two nontrivial countable lattice-ordered groups and obtain, as a consequence of the method, that the free product of nontrivial lattice-ordered groups is directly indecomposable and has trivial center.
\end{abstract}

1. Introduction. The free lattice-ordered group on a countably infinite set of generators was shown to be isomorphic to a doubly transitive sublattice subgroup of the lattice-ordered group of all order-preserving permutations of the rational line (see [1 or 2, Theorem 6.7, and 9]). The proof is easily extended to give a faithful doubly transitive representation for the free lattice-ordered group on any infinite set of generators. More recently, Kopytov [6] and, independently, McCleary [9] proved by a far more subtle technique that the free lattice-ordered group on at least two generators possesses a faithful doubly transitive representation (on the rational line if the number of generators is finite or countable). The ideas were further extended in [3] to prove:

1. If $G$ is any lattice-ordered group and $F$ is a free lattice-ordered group on an infinite set of generators of cardinality at least $|G|$, then the free product (in the category of lattice-ordered groups) of $G$ and $F$ has a faithful doubly transitive representation on some ordered field of cardinality $|F|$.

2. If $G$ is any countable lattice-ordered group and $F$ is a free lattice-ordered group on a finite number $(>1)$ of generators, then the free product (in the category of lattice-ordered groups) of $G$ and $F$ has a faithful doubly transitive representation on the rational line.

Although the proof of 2 is the more delicate, the purpose of this note is to extend it. (In contrast, I have been unable to extend the proof of 1.) Specifically,

THEOREM. The free product (in the category of lattice-ordered groups) of any two nontrivial countable lattice-ordered groups has a faithful doubly transitive representation on the rational line.

Now doubly transitive sublattice subgroups of the lattice-ordered group of all order-preserving permutations of the rational line have trivial center $[\mathbf{2}$, Lemma 1.6.8] and are directly indecomposable (in the category of lattice-ordered groups) [11, Lemma 2]-indeed, in the category of groups [4]. Hence

Received by the editors March 21, 1986.

1980 Mathematics Subject Classification (1985 Revision). Primary 06F15; Secondary 20E06, 20B22.

Research supported in part by NSF grant 8401745 . 
COROLLARY 1. The free product (in the category of lattice-ordered groups) of any two nontrivial countable lattice-ordered groups has trivial center and is directly indecomposable (in this category).

The proof of the theorem, although it does not yield a faithful doubly transitive representation of the free product of arbitrary nontrivial lattice-ordered groups, is adaptable to show:

COROLLARY 1'. The free product (in the category of lattice-ordered groups) of any family of nontrivial lattice-ordered groups has trivial center and is directly indecomposable (in this category or the category of groups).

CAUTION. Corollary $1^{\prime}$ does not follow immediately from Corollary 1 . Indeed, we would like to know if:

Whenever $G_{0}$ and $H_{0}$ are (finitely generated) sublattice subgroups of the latticeordered groups $G$ and $H$ respectively, is the sublattice subgroup of the free product (in the category of lattice-ordered groups) of $G$ and $H$ generated by $G_{0} \cup H_{0}$ isomorphic to the free product (in this category) of $G_{0}$ and $H_{0}$ ?

Powell and Tsinakis [12] proved the direct indecomposability of free products of nontrivial Abelian lattice-ordered groups (in the category of Abelian lattice-ordered groups) and of nontrivial finitely generated lattice-ordered groups belonging to any proper subvariety of lattice-ordered groups (the free product being taken in the variety) [13, Corollary 8.7], but leave open the general problem for free products in the variety of all lattice-ordered groups [13, Problem 10.9]. Corollary $1^{\prime}$ therefore answers their question for the variety of all lattice-ordered groups.

In any doubly transitive sublattice subgroup of the lattice-ordered group of all order-preserving permutations of a totally ordered set, there is a countable (infinite) pairwise disjoint set. Thus

COROLLARY 2. The free product (in the category of lattice-ordered groups) of any two nontrivial countable lattice-ordered groups contains a countable set of pairwise disjoint elements.

This partially answers [13, Problem 10.7].

Any nontrivial lattice-ordered group which has a faithful doubly transitive representation generates the variety of all lattice-ordered groups (see [2, p. 247]). Hence

COROLLARY 3. The free product (in the category of lattice-ordered groups) of any two nontrivial countable lattice-ordered groups generates the variety of all lattice-ordered groups.

This improves Theorem 2.1 and Corollary 2.1.1 of [7]. Again the proof cf Corollary $1^{\prime}$ extends the result to an arbitrary family of nontrivial lattice-ordered groups so that the word "countable" can be omitted from the statement of Corollary 3.

CAUTION. If $G$ and $H$ are arbitrary lattice-ordered groups, then

$$
\left[\left[g \vee e, h^{-1}\left(g^{-1} \vee e\right) h\right],\left[h^{-1}(g \vee e) h, g^{-1} \vee e\right]\right]=e
$$

holds in the free product (in the category of lattice-ordered groups) of $G$ and $H$ for any $g \in G$ and $h \in H$, where $[a, b]=a^{-1} b^{-1} a b$; so if $G$ is not totally ordered and $g \in G$ with $g \nsupseteq e$ and $g \not e$, the subgroup of this free product generated by $G \cup H$ is not the group free product of $G$ and $H$ (see [5]). 
2. Background. We recall some definitions and facts from [2].

A group that is also a lattice which satisfies the identities $x(y \vee z) t=x y t \vee x z t$ and $x(y \wedge z) t=x y t \wedge x z t$ is said to be a lattice-ordered group. Lattice-ordered groups are torsion-free $\left(x^{m}=e \rightarrow x=e\right.$ if $m$ is a positive integer and $e$ denotes the identity element) distributive lattices $(x \wedge(y \vee z)=(x \wedge y) \vee(x \wedge z))$ (see [2, Lemmas 1.11.1 and 1.11.2]). Thus every lattice-ordered group expression $w(\mathbf{x})$ can be rewritten in the form $\bigvee_{i} \bigwedge_{j} w_{i j}$, where $i$ and $j$ range over finite index sets and $w_{i j}$ are just group expressions, say $w_{i j}=x_{i j 0} \cdots x_{i j k}$ with $x_{i j 0}=e$ and each $x_{i j n}$ a generator, the inverse of a generator, or $e$. The set $\left\{x_{i j 0} \cdots x_{i j n}: 0 \leq n \leq k, i \in I\right.$, $j \in J\}$ is called the set of initial subwords of $w$.

Let $\mathbf{R}$ denote the real line and $\Omega \subseteq \mathbf{R}$ with the inherited order. We write $A(\Omega)$ for the lattice-ordered group of all order-preserving permutations of $(\Omega, \leq)$. where $f \leq g$ if and only if $\alpha f \leq \alpha g$ for all $\alpha \in \Omega$. By [2, Lemmas 2.5.1 and 2.5.2 and Holland's Theorem (Appendix I)], any countable lattice-ordered group can be embedded (as a lattice-ordered group) in $A(\mathbf{Q})$, where $\mathbf{Q}$ denotes the rational line. A sublattice subgroup $G$ of $A(\Omega)$ is said to be doubly transitive if whenever $\alpha, \beta, \gamma, \delta \in \Omega$ with $\alpha<\beta$ and $\gamma<\delta$, there exists $g \in G$ such that $\alpha g=\gamma$ and $\beta g=\delta$. (Such sublattice subgroups are $m$-transitive for every finite $m$-see $[2$, Lemma 1.10.1].) A lattice-ordered group isomorphic to such a sublattice subgroup is said to have a faithful doubly transitive representation on $\Omega$. Finally, if $f \in A(\Omega)$, then $\operatorname{supp}(f)=\{\alpha \in \Omega: \alpha f \neq \alpha\}$ is called the support of $f$. A doubly transitive sublattice subgroup of $A(\Omega)$ in which no nonidentity element has support bounded both above and below in $\Omega$ is called pathological. Pathological lattice-ordered groups are never completely distributive [8]. It will follow from the proof of the theorem that the representation is pathological and, consequently, free products of nontrivial lattice-ordered groups are never completely distributive.

For any terms and background that I have unwittingly omitted, see [2].

3. Proof of the Theorem. Let $G * H$ denote the free product of $G$ and $H$ in the category of lattice-ordered groups. Since $G$ and $H$ are countable and nontrivial, so is $G * H$. It follows (see $\S 2$ ) that $G * H$ can be embedded in $A(\Delta)$ for any nondegenerate open interval $\Delta$ of the rational line $\mathbf{Q}$. Let $w_{0}, w_{1}, w_{2}, \ldots$ be an enumeration of the nonidentity elements of $G * H$. Let

$$
\begin{array}{cl}
\Delta_{n}=\left(2 n-\frac{1}{9}, 2 n+\frac{10}{9}\right), & \Lambda_{n}=\left(2 n-\frac{1}{3}, 2 n+\frac{4}{3}\right), \\
A_{n, 0}=\left(2 n+\frac{11}{9}, 2 n+\frac{13}{9}\right), & A_{n, 1}=\left(2 n+\frac{14}{9}, 2 n+\frac{16}{9}\right)
\end{array}
$$

and

$$
B_{n}=\left(2 n+\frac{25}{18}, 2 n+\frac{29}{18}\right), \quad n \in\{0,1,2, \ldots\}=\omega
$$

Hence

$$
\Delta_{n} \subseteq \Lambda_{n}, \quad \Lambda_{n}<B_{n}<\Lambda_{n+1}, \quad \Delta_{n}<A_{n, 0}<A_{n, 1}<\Delta_{n+1}
$$

with $A_{n, 0} \cap \Lambda_{n} \neq \varnothing \neq A_{n, 1} \cap \Lambda_{n+1}$, and $A_{n, i} \cap B_{n} \neq \varnothing(i=0,1)$ for all $n \in \omega$.

Embed $G * H$ in $A\left(\Delta_{n}\right)$ by $\phi_{n}$, say $(n \in \omega)$. Clearly we may assume that for some $\alpha_{n} \in \Delta_{n}$ with $2 n \leq \alpha_{n}<2 n+1$,

(1) $\alpha_{n} w_{n} \neq \alpha_{n}, 2 n=\min \left\{\alpha_{n}\left(u \phi_{n}\right): u\right.$ initial subword of $\left.w_{n}\right\}$ and $2 n+1=$ $\max \left\{\alpha_{n}\left(u \phi_{n}\right): u\right.$ initial subword of $\left.w_{n}\right\}$. 
For each $n \in \omega$, define $\theta_{n}: H \rightarrow A\left(\Lambda_{n}\right)$ as follows:

(a) If every element of $H \phi_{n}$ fixes $2 n$ and $2 n+1$, let $g_{n, 0}, g_{n, 1} \in G$ be such that $(2 n)\left(g_{n, 0} \phi_{n}\right) \leq 2 n$ and $(2 n+1)\left(g_{n, 1} \phi_{n}\right)>(2 n+1)$. (Since $2 n=\alpha_{n}\left(u_{0} \phi_{n}\right)$ and $2 n+1=\alpha_{n}\left(u_{1} \phi_{n}\right)$ for some initial subwords $u_{0}, u_{1}$ of $w_{n}$, such elements of $G$ must exist.) Let $\theta_{n, i}(i=0,1)$ be embeddings of $H$ in $A\left(\left(2 n-\frac{1}{3}, 2 n\right)\right)$ and $A\left(\left(2 n+1,2 n+\frac{4}{3}\right)\right)$ respectively such that

$$
(2 n)\left(g_{n, 0} \phi_{n}\right)\left(h_{n, 0} \theta_{n, 0}\right)=2 n-\frac{2}{9} \quad \text { and } \quad(2 n+1)\left(g_{n, 1} \phi_{n}\right)\left(h_{n, 1} \theta_{n, 1}\right)=2 n+\frac{11}{9}
$$

for some $h_{n, 0}, h_{n, 1} \in H$. If $h \in H$, let

$$
\lambda\left(h \theta_{n}\right)= \begin{cases}\lambda\left(h \phi_{n}\right) & \text { if } \lambda \in[2 n, 2 n+1], \\ \lambda\left(h \theta_{n, 0}\right) & \text { if } \lambda \in\left(2 n-\frac{1}{3}, 2 n\right), \\ \lambda\left(h \theta_{n, 1}\right) & \text { if } \lambda \in\left(2 n+1,2 n+\frac{4}{3}\right) .\end{cases}
$$

(b) If every element of $H \phi_{n}$ fixes $2 n$ but for some element $h_{n, 1} \in H$, $2 n+1<(2 n+1)\left(h_{n, 1} \phi_{n}\right)$, let $a_{n} \in A\left(\Lambda_{n}\right)$ be the identity on $\left(2 n-\frac{1}{3}, 2 n+1\right]$ with $2 n+\frac{11}{9}=(2 n+1)\left(h_{n, 1} \phi_{n}\right) a_{n}$. For $h \in H$, let

$$
\lambda\left(h \theta_{n}\right)= \begin{cases}\lambda\left(a_{n}^{-1}\left(h \phi_{n}\right) a_{n}\right) & \text { if } \lambda \in\left[2 n, 2 n+\frac{4}{3}\right), \\ \lambda\left(h \theta_{n, 0}\right) & \text { if } \lambda \in\left(2 n-\frac{1}{3}, 2 n\right),\end{cases}
$$

where $\theta_{n, 0}$ is defined as in (a).

(c) If every element of $H \phi_{n}$ fixes $2 n+1$ but for some element $h_{n, 0} \in H$, $(2 n)\left(h_{n, 0} \phi_{n}\right)<2 n$, proceed dually to (b).

(d) If for some $h_{n, 0}, h_{n, 1} \in H,(2 n)\left(h_{n, 0} \phi_{n}\right)<2 n$ and $2 n+1<(2 n+1)\left(h_{n, 1} \phi_{n}\right)$, let $a_{n} \in A\left(\Lambda_{n}\right)$ be the identity on $[2 n, 2 n+1]$ with $(2 n)\left(h_{n, 0} \phi_{n}\right) a_{n}=2 n-\frac{2}{9}$ and $2 n+\frac{11}{9}=(2 n+1)\left(h_{n, 1} \phi_{n}\right) a_{n}$. Let $h \theta_{n}=a_{n}^{-1}\left(h \phi_{n}\right) a_{n}$ for all $h \in H$.

Note that in all cases $\theta_{n}$ is an embedding and that whereas $\operatorname{supp}\left(g \phi_{n}\right) \subseteq \Delta_{n}$ for all $g \in G, \operatorname{supp}\left(h \theta_{n}\right)$ overspills into $\Lambda_{n} \backslash \Delta_{n}$ at both ends for appropriate elements of $H \theta_{n}$, namely $h_{n, 0} \theta_{n}$ and $h_{n, 1} \theta_{n}$.

Let $\phi_{n, i}: G \rightarrow A\left(A_{n, i}\right)(i=0,1)$ be embeddings such that

and

$$
\left(2 n+\frac{11}{9}\right)\left(h_{n, 1} \theta_{n}\right)\left(\bar{g}_{n, 0} \phi_{n, 0}\right)=2 n+\frac{25}{18}
$$

$$
\left(2 n+\frac{16}{9}\right)\left(h_{n+1,0} \theta_{n+1}\right)\left(\bar{g}_{n, 1} \phi_{n, 1}\right)=2 n+\frac{29}{18}
$$

for some $\bar{g}_{n, 0}, \bar{g}_{n, 1} \in G$, and let $\bar{\phi}_{n}: G \rightarrow A\left(\left(2 n-\frac{1}{3}, 2 n+\frac{5}{3}\right)\right)$ be given by

$$
\beta\left(g \bar{\phi}_{n}\right)= \begin{cases}\beta\left(g \phi_{n}\right) & \text { if } \beta \in \Delta_{n}, \\ \beta\left(g \phi_{n, i}\right) & \text { if } \beta \in A_{n, i}(i=0,1), \\ \beta & \text { otherwise. }\end{cases}
$$

Then $\bar{\phi}_{n}$ is an embedding of $G$ into $A\left(\left(2 n-\frac{1}{3}, 2 n+\frac{5}{3}\right)\right)$.

Let $\hat{\theta}_{n}: H \rightarrow A\left(B_{n}\right)$ be any embedding such that

$$
\left(2 n+\frac{25}{18}\right)\left(\bar{g}_{n, 0} \phi_{n, 0}\right)\left(h_{n} \hat{\theta}_{n}\right)=\left(2 n+\frac{29}{18}\right)\left(\bar{g}_{n, 1} \phi_{n, 1}\right)
$$

for some $h_{n} \in H$, and define $\bar{\theta}_{n}: H \rightarrow A\left(\left(2 n-\frac{1}{3}, 2 n+\frac{5}{3}\right)\right)$ by

$$
\beta\left(h \bar{\theta}_{n}\right)= \begin{cases}\beta\left(h \hat{\theta}_{n}\right) & \text { if } \beta \in B_{n} \\ \beta\left(h \theta_{n}\right) & \text { if } \beta \in \Lambda_{n} \\ \beta & \text { otherwise. }\end{cases}
$$

Then $\bar{\theta}_{n}$ is an embedding. 
Let $\bar{\alpha}_{0}=\inf \left\{-\frac{2}{9}\left(h \bar{\theta}_{0}\right): h \in H\right\} \in \mathbf{R}$; so $\bar{\alpha}_{0} \geq-\frac{1}{3}$. Let $\hat{\phi}_{0}$ be an embedding of $G$ into $A(\mathbf{Q})$ such that if $\Gamma=\left\{-\frac{2}{9}\left(h_{0,0} \bar{\theta}_{0}\right)\left(g \hat{\phi}_{0}\right): g \in G\right\}$, then $\sup \Gamma=-\frac{2}{9}$ and $\inf \Gamma=\bar{\alpha}_{0}$.

We now define maps of $G$ and $H$ into $A\left(\Omega_{0}\right)$, where $\Omega_{0}=\left\{\alpha \in \mathbf{Q}: \alpha>\bar{\alpha}_{0}\right\}$. For $g \in G$, let

$$
\beta(g \phi)= \begin{cases}\beta\left(g \bar{\phi}_{n}\right) & \text { if } \beta \in\left(2 n-\frac{1}{3}, 2 n+\frac{5}{3}\right) \text { for some } n \in \omega \backslash\{0\}, \\ \beta\left(g \bar{\phi}_{0}\right) & \text { if } \beta \in\left(-\frac{2}{9}, \frac{5}{3}\right) \\ \beta\left(g \hat{\phi}_{0}\right) & \text { if } \beta \in\left(\bar{\alpha}_{0},-\frac{2}{9}\right) .\end{cases}
$$

Then $\phi$ is a well-defined homomorphism since $\hat{\phi}_{0}$ and each $\bar{\phi}_{n}$ is. For $h \in H$, let

$$
\beta(h \theta)= \begin{cases}\beta\left(h \bar{\theta}_{0}\right) & \text { if } \beta \in\left(2 n-\frac{1}{3}, 2 n+\frac{5}{3}\right) \text { for some } n \in \omega \backslash\{0\}, \\ \beta\left(h \bar{\theta}_{0}\right) & \text { if } \beta \in\left(\bar{\alpha}_{0}, \frac{5}{3}\right) .\end{cases}
$$

Again $\theta$ is a well-defined homomorphism. Then $(\phi, \theta)$ extends to a homomorphism $\psi$ of $G * H$ into $A\left(\Omega_{0}\right)$. Observe that $\psi\left|\Delta_{n}=\phi_{n}\right| \Delta_{n}$ for each $n \in \omega$ by the definitions of $\phi, \theta$, and $\psi$. Let $T=\left\{\alpha_{0}(f \psi): f \in G * H\right\} \subseteq \Omega_{0}$. By the construction, $\alpha_{n} \in T$ for all $n \in \omega$, and as each $w \in(G * H) \backslash\{e\}$ is $w_{n}$ for some $n \in \omega$ and $\alpha_{n}\left(w_{n} \psi\right)=\alpha_{n}\left(w_{n} \phi_{n}\right) \neq \alpha_{n}$, the natural map $\bar{\psi}$ obtained from $\psi$ by restricting each $f \psi$ to $T$ is an isomorphism of $G * H$ onto a transitive sublattice subgroup of $A(T)$. Since every element of $G \bar{\psi}$ fixes $-\frac{2}{9} \in T$ but $\sup \Gamma=-\frac{2}{9}$ and $\inf \Gamma=\bar{\alpha}_{0}$, the $(G * H) \bar{\psi}$ stabilizer of $-\frac{2}{9}$ has exactly one orbit in $T$ below $-\frac{2}{9}$. Thus $(G * H) \bar{\psi}$ is a doubly transitive sublattice subgroup of $A(T)$ (see [2, Lemma 1.10.1]). Now $T$ is a countable subset of $\mathbf{Q}$ and $A(T)$ is doubly transitive (since $(G * H) \bar{\psi}$ is). It follows that $T$ must be order-isomorphic to the rational line, which proves the theorem.

Observe that if $e \neq w \in G * H$, then as $w, w^{2}, w^{3}, \ldots$ are all distinct, $X=$ $\left\{n \in \omega: w_{n}=w^{m}\right.$ for some $\left.0<m \in \omega\right\}$ is cofinal in $\omega$. For each $n \in X$, $\alpha_{n}\left(w_{n} \bar{\psi}\right)=\alpha_{n}\left(w_{n} \psi\right) \neq \alpha_{n}$, whence $\alpha_{n}(w \bar{\psi}) \neq \alpha_{n}$ for each $n \in X$. Consequently the support of $w$ is not bounded above in $T$; that is, $(G * H) \bar{\psi}$ is pathological. (Aliter: In the enumeration of $G * H$, repeat each word infinitely often.)

4. The general case; Proof of Corollary $\mathbf{1}^{\prime}$. Clearly it is enough to prove Corollary $1^{\prime}$ for the free product of two nontrivial lattice-ordered groups, say $G$ and $H$. Let $\left\{G_{i}: i \in I\right\}$ and $\left\{H_{j}: j \in J\right\}$ enumerate all the nontrivial countable (or just finitely generated) sublattice subgroups of $G$ and $H$ respectively and totally order $I \times J$. Let $\left\{w_{n i j}: n=1,2,3, \ldots\right\}$ enumerate the nonidentity elements of the sublattice subgroup of $G * H$ generated by $G_{i} \cup H_{j}(i \in I, j \in J)$. There is a totally ordered field $\Omega$ containing isomorphic subintervals $\Omega_{i j}$ (with $\Omega_{i_{1} j_{1}}<\Omega_{i_{2} j_{2}}$ if $\left(i_{1}, j_{1}\right)<\left(i_{2}, j_{2}\right)$ in the total order on $\left.I \times J\right)$ such that $G * H$ can be embedded in the lattice-ordered group $A\left(\Omega_{i j}\right)=\operatorname{Aut}\left(\left\langle\Omega_{i j}, \leq\right\rangle\right)$ for each $(i, j) \in I \times J$. As above, for each $(i, j) \in I \times J$, we can find bounded intervals $\Omega_{n i j}$ of $\Omega_{i j}$ with $\Omega_{1 i j}<\Omega_{2 i j}<\cdots$ and points $\alpha_{n i j} \in \Omega_{n i j}$ such that $\alpha_{n i j} w_{n i j} \neq \alpha_{n i j}$ and if $w_{n i j}=\bigvee_{m} \bigwedge_{k} w_{k m n i j}$ with $w_{k m n i j}$ group words, then $\alpha_{n i j} u_{k m n i j} \in \Omega_{n i j}$ for all initial subwords $u_{k m n i j}$ of $w_{k m n i j}$. Indeed, just as in the previous section, we can map $G$ and $H$ into $A\left(\Omega_{i j}\right)$ so that the extension $\phi_{i j}$ to $G * H$ gives $(G * H) \phi_{i j}$ doubly transitive on the orbit $\Delta_{i j}$ of $\alpha_{i j}$ and $w_{n i j} \phi_{i j} \neq e$ for $n=1,2,3, \ldots$, for each $(i, j) \in I \times J$; moreover, $\alpha_{n i j} \in \Delta_{i j}(n=1,2, \ldots,(i, j) \in I \times J)$. Since each $w \in(G * H) \backslash\{e\}$ belongs to 
the sublattice subgroup generated by $G_{i} \cup H_{j}$ for some $i \in I$ and $j \in J$, we obtain an embedding of $G * H$ into $A(\Delta)$, where $\Delta=\bigcup\left\{\Delta_{i j}:(i, j) \in I \times J\right\}$ with the inherited linear ordering.

Now if $G * H=A \times B$ with $A$ and $B$ nontrivial, let $e \neq a \in A$ and $e \neq b \in B$. Let $i \in I$ and $j \in J$ be such that $a$ and $b$ belong to the sublattice subgroup of $G * H$ generated by $G_{i} \cup H_{j}$. By the construction, $a \phi_{i j} \neq e \neq b \phi_{i j}$. But $(G * H) \phi_{i j}=A \phi_{i j} \times B \phi_{i j}$. Since $(G * H) \phi_{i j}$ is doubly transitive on $\Delta_{i j}$, this is impossible (see [4]). Hence $G * H$ is directly indecomposable even in the category of groups.

If $e \neq w \in G * H$, then for some $(i, j) \in I \times J, w \phi_{i j} \neq e$. Since $(G * H) \phi_{i j}$ is doubly transitive on $\Delta_{i j}$, there is $u \in G * H$ such that $\left[w \phi_{i j}, u \phi_{i j}\right] \neq e[\mathbf{2}$, Lemma 1.6.9]. Consequently, $[w, u] \neq e$; so $w$ does not lie in the center of $G * H$. Therefore $G * H$ has trivial center.

5. Concluding remarks. Many of the results on free lattice-ordered groups to be found in [10] hold for free products of countable lattice-ordered groups by the same methods (only the doubly transitive representation is required). Rather than bore the reader, we refer him to $[10$, Corollaries $2,3,4,6,7,8$, and 9 , Proposition 13 and, if $G$ and $H$ have strong order units (e.g., if they are finitely generated), Lemma 14(a), Theorem 15, and Corollary 16] for statements and proofs.

\section{BIBLIOGRAPHY}

1. A. M. W. Glass, l-simple lattice-ordered groups, Proc. Edinburgh Math. Soc. 19 (1974), 133138.

2. Univ. Press, 1981.

3. __ Effective embeddings of countable lattice-ordered groups, Proc. 1st Internat. Sympos. on Ordered Algebraic Structures, Luminy-Marseilles, 1984 (S. Wolfenstein, Ed.), Research \& Exposition in Math., 14, Heldermann-Verlag, pp. 63-69.

4. A. M. W. Glass and J. S. Wilson, Normal subgroups with non-trivial centraliser in doubly homogeneous permutation groups (unpublished manuscript).

5. W. C. Holland, Group equations which hold in lattice-ordered groups, Symposia Math. 21 (1977), 365-378.

6. V. M. Kopytov, Free lattice-ordered groups, Siberian Math. J. 24 (1983), 98-101 (English translation).

7. J. Martinez, Free products in varieties of lattice-ordered groups, Czechoslovak Math. J. 22 (97) (1972), 535-553.

8. S. H. McCleary, o-2-transitive ordered permutation groups, Pacific J. Math. 49 (1973), 425-429.

9. __ Free lattice-ordered groups, Ordered Algebraic Structures (W. B. Powell and C. Tsinakis, eds.), Lecture Notes in Pure and Applied Math., vol. 99, Marcel Dekker, New York, 1985, pp. $139-154$.

10. _ Free lattice-ordered groups represented as o-2-transitive l-permutation groups, Trans. Amer. Math. Soc. 290 (1985), 69-79.

11. N. Ya. Medvedev, Decomposition of free l-groups into l-direct products, Siberian Math. J. 21 (1980), 691-696 (English translation).

12. W. B. Powell and C. Tsinakis, Free products of abelian l-groups are cardinally indecomposable, Proc. Amer. Math. Soc. 86 (1982), 385-390.

13. _ Free products of lattice-ordered groups, Algebra Universalis 18 (1984), 178-198.

Department of Mathematics, Bowling Green State University, Bowling GREEN, OHIO 43403 